\title{
ELETRODOS DE PASTA DE CARBONO MODIFICADOS COM ÁCIDOS HÚMICOS: ESTUDO E DETERMINAÇÃO DE METAIS EM MEIO AQUOSO
}

\author{
Frank Nelson Crespilho e Maria Olímpia Oliveira Rezende* \\ Instituto de Química de São Carlos, Universidade de São Paulo, Av. Trabalhador Sãocarlense, 400, 13560-970 São Carlos - SP \\ Recebido em 19/9/03; aceito em 14/5/04; publicado na web em 09/08/04
}

\begin{abstract}
CARBON PASTE ELECTRODES MODIFIED WITH HUMIC ACIDS: STUDY AND DETERMINATION OF METALS IN AQUEOUS SOLUTION. Since 1992, the carbon paste electrodes modified with humic acids have been used for studying the behavior of metals in aqueous solutions. Many parameters influence the performance of the electrodes, such as the humic acid ratio, the nature of the humic acid, the accumulation time, the $\mathrm{pH}$, the scan rate, and the preparation of the electrodes itself. There are various methos of preparing the electrodes. The goal of this paper is to review some of them. The advantages of using electrodes modified with humic acids as electrochemical sensors for evaluating metals in aqueous solution are stressed.
\end{abstract}

Keywords: humic acid; modified electrode; metals.

\section{INTRODUÇÃO}

\section{Breve histórico sobre os eletrodos de pasta de carbono modificados}

A Química Analítica vem aperfeiçoando cada vez mais os métodos e técnicas que possam determinar quantidades muito pequenas de analitos em soluções aquosas. As técnicas eletroanalíticas, principalmente aquelas em que a varredura de potenciais gera sinais analíticos na forma de correntes, têm sido empregadas na determinação de diversos analitos ${ }^{1}$. Dentre as várias técnicas, algumas destacamse por apresentar grande sensibilidade e reprodutibilidade dos sinais analíticos. Como exemplos, tem-se a voltametria de redissolução anódica e catódica ${ }^{2}$, voltametria de pulso diferencial ${ }^{2}$ e a voltametria de onda quadrada, sendo que essa última vem ganhando a atenção da comunidade científica devido a sua versatilidade na caracterização das espécies eletroativas ${ }^{3}$.

Com o advento das técnicas eletroanalíticas, os sensores eletroquímicos ganharam novos espaços nas diversas áreas de pesquisas. Dessa forma, vários trabalhos vêm sendo publicados em revistas especializadas, em que os autores relatam diferentes tipos de eletrodos utilizados na quantificação de espécies eletroativas ${ }^{3-7}$. Podese destacar entre eles os eletrodos de pasta de carbono (EPCs) ${ }^{8-12}$.

O primeiro EPC foi desenvolvido por Adams, com intuito de substituir o eletrodo gotejante de mercúrio. Seu objetivo era atingir potenciais mais positivos, nos quais o eletrodo de mercúrio não podia ser aplicado ${ }^{13}$. Os primeiros trabalhos de Adams visaram a caracterização do EPC, aplicando técnicas básicas em eletroquímica ${ }^{14}$. Em 1963, ele utilizou pela primeira vez o EPC para determinar ouro e prata, utilizando a voltametria de redissolução anódica ${ }^{15}$.

A partir de 1964, French e Kuwana trabalharam com líquidos orgânicos e investigaram o comportamento eletroquímico desses compostos incorporados à pasta de carbono ${ }^{16}$. A partir desse momento, surge o conceito de "agente modificador", que pode ser definido como qualquer espécie química com propriedades de melhorar a sensibilidade e a estabilidade de um EPC. Em 1965, Kuwana e Schultz publicaram um artigo envolvendo EPC e compostos orgâni$\cos ^{17}$. Assim, sendo os pioneiros na área, abriram caminho para no-

\footnotetext{
*e-mail: mrezende@iqsc.usp.br
}

vos pesquisadores utilizarem substâncias eletroquimicamente ativas como modificadores ${ }^{8}$.

Com o avanço das pesquisas em eletroanálise, estudos têm sido realizados com o intuito de se obter melhores sensores aplicados à determinação de compostos em solução, fato esse que pode ser confirmados pelos inúmeros trabalhos publicados na década de $70^{18}$.

Os primeiros pesquisadores a introduzir uma substância que continha um grupo funcional complexante em um EPC foram Chek e Nelson, em $1978^{19}$. O objetivo dos autores era a imobilização, por pré-concentração, de prata na superfície do eletrodo.

Em 1981, um método de mistura direta do agente modificador à pasta de carbono foi proposto por Ravichandran e Baldwin ${ }^{20}$. Nesse trabalho, eles descreveram a facilidade em adicionar o agente modificador à pasta de carbono, fato que chamou a atenção dos cientistas da época e incentivou o uso desse método, que até os dias atuais é muito empregado. O trabalho "Chemically Modified Carbon Paste Electrodes” publicado por eles em 1981 possui atualmente setenta e cinco citações cadastradas na ScientificDirect ${ }^{18}$, sendo referenciado até os dias atuais ${ }^{21}$. Dessa forma, vários tipos de sensores baseados em eletrodos de pasta de carbono modificados (EPCM) ganharam espaço em eletroquímica e novos artigos, dessa vez em grande número, começaram a ser publicados em revistas especializadas ${ }^{22-27}$. Assim, a partir de 1981 até os dias atuais, os temas dos trabalhos diversificaram-se, como por exemplo, EPCM aplicado à determinação de prata e cobre(II) ${ }^{23}$; modificação do EPC com enzimas para determinação de glicose $^{24}$; EPCM aplicado em eletrocatálise ${ }^{25}$; determinação de titânio $(\mathrm{IV})^{26}$; EPC modificado com produtos naturais para a determinação de fármacos ${ }^{27}$; EPC modificados com zeólitas para a determinação de cobre $^{28}$; EPC modificados com ácidos húmicos para estudo com metais e pesticidas ${ }^{9-12}$, entre outros.

A partir de 1992, alguns pesquisadores descobriram grandes vantagens em se modificar um EPC com ácidos húmicos $(\mathrm{AH})^{9-12}$. Para entender o porquê de se modificar um EPC com $\mathrm{AH}$, é preciso compreender as características estruturais e a reatividade dessas moléculas.

\section{VANTAGENS DA UTILIZAÇÃO DE ÁCIDOS HÚMICOS COMO MODIFICADORES EM EPCs}

Embora não haja um modelo definido sobre a estrutura dos $\mathrm{AH}^{29-31}$, existe um fato que muito interessa àqueles que trabalham com 
eletroanalítica: os AH são compostos com grande capacidade de complexação de metais ${ }^{9-11,32-34}$ e possuem grande afinidade por compostos orgânicos ${ }^{11,12,35}$.

Os AH são os principais constituintes do húmus natural. Podem ser extraídos de turfeiras (material geológico rico em matéria orgânica), vermicompostos e de sistemas aquáticos ${ }^{9-12}$. Ao avaliar a estrutura dos $\mathrm{AH}$ por espectrometria de massas acoplada a um pirolisador, uma maior predominância de grupos alifáticos foi observada por Schulten e Schnitzer ${ }^{30}$. A Figura 1 apresenta alguns grupamentos que estão presentes na estrutura do $\mathrm{AH}$ proposta por Schulten e Schnitzer.
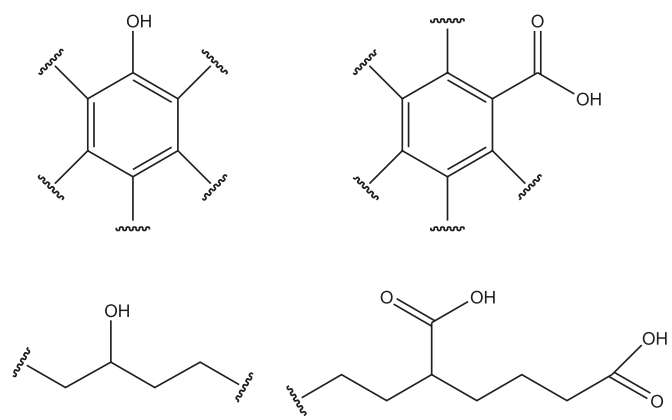

Figura 1. Alguns grupamentos presentes na estrutura de uma molécula de AH proposta por Schulten e Schnitzer (fonte: ref. 30)

Vale salientar que há outros modelos propostos na literatura ${ }^{29,31}$.

A reatividade das substâncias húmicas está relacionada, principalmente, com a grande quantidade de grupos funcionais como $\mathrm{COOH}, \mathrm{OH}$-fenólicos, $\mathrm{OH}$-enólicos, $\mathrm{OH}$-alcoólicos, $\mathrm{C}=\mathrm{O}$ de quinonas e hidroxiquinonas, e cetonas ${ }^{32,33}$.

As características físicas e químicas de $\mathrm{AH}$ extraídos de diferentes localidades dependem de muitos fatores, dentre os quais podemos citar a procedência do material, condições climáticas (zona tropical, subtropical, moderada ou fria), acidez e grau de umidade do $\operatorname{solo}^{30,32,33}$. A Sociedade Internacional de Substâncias Húmicas (IHSS) recomenda alguns procedimentos para a extração e purificação de $\mathrm{AH}^{36}$.

Assim, pode-se construir um EPC modificado com ácidos (EPC$\mathrm{AH}$ ) com intuito de aproveitar as características dessas moléculas, por exemplo, na complexação de metais. Os EPC-AHs representam uma grande ferramenta em estudos analíticos e ambientais, que serão mostrados ao longo desta revisão. A construção e a otimização do EPC-AH exige diversas etapas, que serão relatadas a seguir.

\section{A BUSCA PELA OTIMIZAÇÃO DO EPC-AH}

O primeiro EPC-AH construído e relatado na literatura não continha $\mathrm{AH}$ purificado em sua composição ${ }^{37}$. A modificação da superfície eletródica foi realizada com turfa, material rico em $\mathrm{AH}$. A desvantagem desse tipo de procedimento está na incorporação indireta de outras substâncias, como sílica e sais inorgânicos entre outros compostos presentes na turfa, que podem atrapalhar o sinal de resposta. Além disso, o eletrodo pode tornar-se muito resistivo pelo fato de conter compostos não condutores, resultando em perda de sensibilidade $^{1,2}$. Por fim, as turfas contêm vários metais complexados com os $\mathrm{AH}^{32,35,36}$ que podem interferir nos resultados.

Apesar dessas desvantagens, Wang publicou um trabalho em 1992 no qual descreve algumas vantagens da modificação da pasta, obtendo resultados satisfatórios ${ }^{37}$. $\mathrm{O}$ autor concluiu que ao modificar o EPC com $15 \%(\mathrm{~m} / \mathrm{m})$ de turfa, o EPC-AH torna-se mais sensível no processo de pré-concentração de cobre(II) na superfície do eletrodo. Nesse mesmo ano, Navratilova e Kula ${ }^{38}$ quiseram demonstrar a seletividade do EPC-AH na detecção de mercúrio(II) e chegaram à conclusão que esse tipo de eletrodo não se comporta como eletrodo íon seletivo para esse metal. Eles obtiveram bons resultados no que se refere ao aumento de sensibilidade ${ }^{38}$. Mais adiante, esses autores verificaram que o EPC-AH era capaz de determinar vários íons em solução, como por exemplo, cobre (II), zinco (II) e prata (I) ${ }^{39}$.

Em 1994, Jeong et al. realizaram o primeiro trabalho de quantificação de cobre (II) utilizando EPC-AH ${ }^{40}$. Esse trabalho abriu as portas para um novo conceito em utilizar o EPC-AH, evitando o "efeito memória". A renovação da superfície era realizada antes de cada determinação. Porém, esse trabalho ainda não apresentava as condições analíticas ideais. $\mathrm{O}$ fato se explica devido ao longo tempo de análise e às várias etapas de pré-concentração de cobre (II) na superfície do eletrodo. A pré-concentração era realizada por 3 min e repetida 10 vezes antes de se fazer a varredura de potencial. Outro inconveniente era que as varreduras não eram realizadas na mesma cela eletroquímica na qual o analito se encontrava. Após a etapa de pré-concentração, as correntes de pico eram obtidas em outra cela contendo eletrólito suporte livre de íons cobre, fazendo com que a análise levasse mais tempo ${ }^{40}$.

Os primeiros trabalhos realizados no Brasil envolvendo EPCAH foram desenvolvidos por Silva e Rezende, por volta de $1995^{41}$. Os autores utilizaram EPC modificados com AH comerciais e extraídos de turfas, sendo esses purificados antes da incorporação à pasta. Eles conseguiram otimizar a preparação dos eletrodos, de tal forma que a eletroanálise leva pouco menos de $7 \mathrm{~min}$. Além disso, o eletrodo era constituído de apenas $3 \%(\mathrm{~m} / \mathrm{m})$ de $\mathrm{AH}$ e vários métodos de homogeneização da pasta foram testados com sucesso ${ }^{10}$.

Baseado nos resultados iniciais obtidos por Silva e Rezende ${ }^{10}$, outros trabalhos começaram a ser realizados, utilizando EPC-AH em estudos com pesticidas ${ }^{11,12}$ e também na determinação de cobre (II) em amostras de aguardente de cana?

\section{MÉTODOS DE PREPARAÇÃO DO EPC-AH}

De acordo com a literatura, os métodos de preparação dos EPCAHs variam e podem ser otimizados segundo a necessidade de cada determinação ${ }^{9-12,37-40}$. Pode-se utilizar o método de adsorção, homogeneização com hexano via ultra-som, ou de adição direta do AH na pasta ${ }^{10,39,41}$.

O método de adição direta consiste em adicionar certa massa de AH em uma certa porção de grafite em pó e um agente aglutinante. O agente aglutinante pode ser constituído por óleo vegetal ou mineral, sendo que os mais comumente usados são nujol e óleo de rícino. A função do agente aglutinante é transformar a mistura de pó de grafite e o agente modificador $(\mathrm{AH})$ em uma pasta compacta e consistente. É importante salientar que a massa excessiva de óleo pode contribuir fortemente para o aumento da resistividade do eletrodo. Com relação à adição de $\mathrm{AH}$ na pasta, a literatura registra proporções variadas, desde 3 até $20 \%(\mathrm{~m} / \mathrm{m})^{9-12,37-40}$. Após a adição das substâncias que compõem a pasta, esta deve ser homogeneizada. Essa operação pode ser feita em um almofariz com pistilo durante $15 \mathrm{~min}$.

No método de adsorção, o EPC é imerso em uma solução de AH por um determinado tempo. $\mathrm{O} \mathrm{AH}$ adsorve-se na superfície do eletrodo, que pode ser utilizado como eletrodo modificado na determinação das espécies ${ }^{41}$. Porém, essa técnica não é reprodutível, pois a superfície não fica homogênea e pode-se desprender AH durante as determinações ${ }^{41}$.

Quando se utiliza ultra-som, prepara-se uma solução de AH em n-hexano. A mistura fica sob ação do ultra-som até a evaporação. Em seguida, adiciona-se o óleo e homogeniza-se a pasta durante $2 \mathrm{~h}^{39}$.

Embora a adição direta seja um procedimento simples e satisfatório, alguns experimentos foram realizados com intuito de 
aumentar a homogeneidade da pasta, tornando, assim, mais reprodutíveis as determinações. Silva relata que uma préhomogeneização do pó grafite com AH em solução de hidróxido de lítio sob agitação, seguida de liofilização, melhora a reprodutibilidade dos resultados ${ }^{41}$. Na Figura 2, apresenta-se esquematicamente o método de preparação do EPC e do EPC-AH proposto por Silva ${ }^{10}$.

Eletrodo de Pasta de Carbono

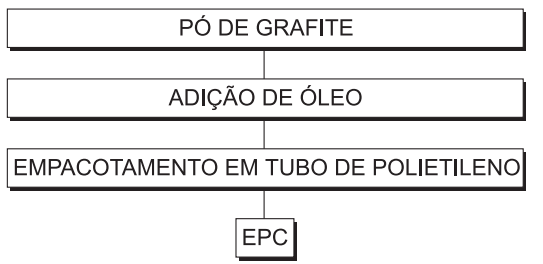

Eletrodo de Pasta de Carbono Modificado

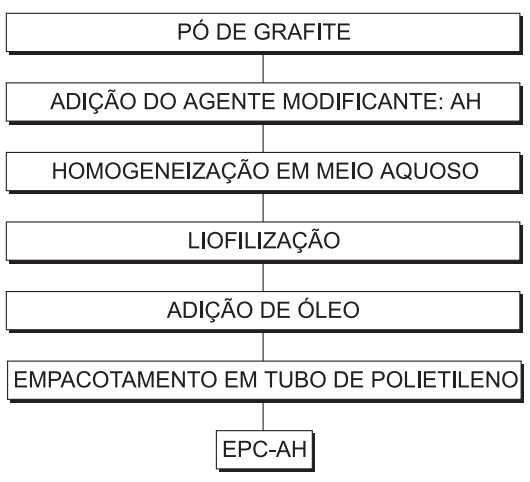

Figura 2. Esquema da preparação do EPC e do EPC-AH (fonte: ref. 9)

Os modelos de preparação do EPC-AH geralmente utilizam o empacotamento da pasta em tubos de vidro e polietileno ${ }^{9-12,37-40}$. O contato elétrico pode ser uma haste de metal condutor ${ }^{9}$. A Figura 3 apresenta as características do EPC-AH.

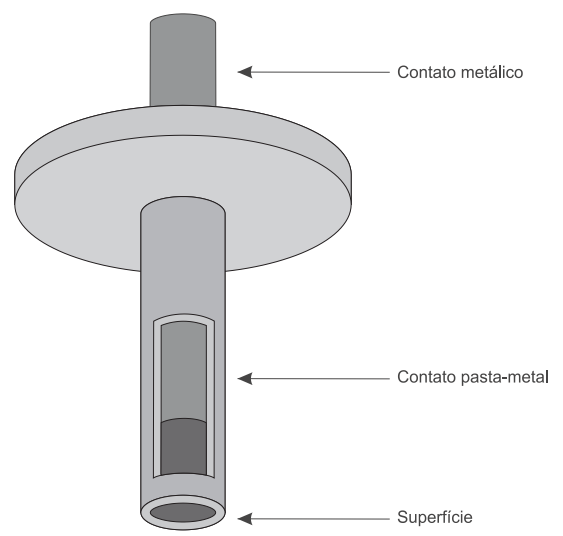

Figura 3. Desenho ilustrativo de um EPC-AH

Além de fornecer o contato elétrico entre a pasta e a fonte de potencial, a haste de metal tem a função de pressionar a pasta para que a superfície possa ser renovada ${ }^{9}$. Geralmente, a renovação é feita friccionando-se o eletrodo em uma folha de papel limpo ${ }^{9-12}$.

\section{TÉCNICAS ELETROANALÍTICAS UTILIZANDO EPC-AH}

Dentre as técnicas utilizadas em investigações eletroquímicas, as de varredura linear de potencial são as mais citadas em estudos de
EPC-AH e a voltametria cíclica é a mais empregada ${ }^{9-12,40-45}$. Por meio dela, pode-se averiguar vários fenômenos de superfície e caracterizá$\operatorname{los}^{46}$. Isso vem ocorrendo porque a maioria dos trabalhos envolvendo EPC-AH estão em fase inicial de estudo e existe a necessidade de entendimento dos fenômenos que ocorrem no sistema eletrodoeletrólito. Técnicas mais sensíveis podem ser utilizadas, como exemplo a voltametria de pulso, voltametria de pulso diferencial e a voltametria de onda quadrada $3,38,41,46$.

A maioria das determinações utilizando EPC-AH tiveram como objetivo a determinação de metais em solução e o estudo da interação entre os metais e os $\mathrm{AH}^{10,40-45}$. Porém, devido à grande capacidade de interação de $\mathrm{AH}$ com outros compostos (pesticidas, por exemplo), a utilização de EPC-AH vem crescendo ${ }^{11,12}$.

Uma ferramenta importante para pré-concentrar um analito na superfície do eletrodo e estudar o comportamento eletroquímico das espécies frente aos AH é a Voltametria de Redissolução, que se divide em Anódica (VRA) e Catódica (VRC). Na VRC aplica-se um potencial suficientemente positivo para que as espécies eletroativas sejam oxidadas na superfície do EPC-AH. Após a pré-concentração, o potencial é varrido no sentido inverso e as espécies são reduzidas. A corrente de pico é registrada como um sinal analítico. Na VRA ocorre o inverso ${ }^{9-12}$.

Considerando-se um estudo envolvendo EPC-AH e cobre(II) ${ }^{9}$, a VC de Redissolução Anódica (VCRA) do cobre utilizando EPC-AH envolve a pré-concentração do metal na superfície do eletrodo, pela aplicação de um potencial suficientemente negativo para que o cobre se reduza na superfície do eletrodo. Em seguida, a varredura de potencial é realizada no sentido anódico, oxidando o cobre que ficara depositado anteriormente. O ciclo é completado por uma varredura no sentido catódico, voltando ao potencial inicial. A corrente registrada na etapa anódica é utilizada como sinal analítico, uma vez que a corrente de pico será proporcional à concentração da espécie em solução. Para aumentar o efeito de acumulação, a solução pode ser agitada e a quantidade depositada também aumenta com o tempo de deposição. A Figura 4 mostra de maneira esquemática como ocorre o processo de VCRA com cobre (II), em sua etapa anódica ${ }^{9,10}$.
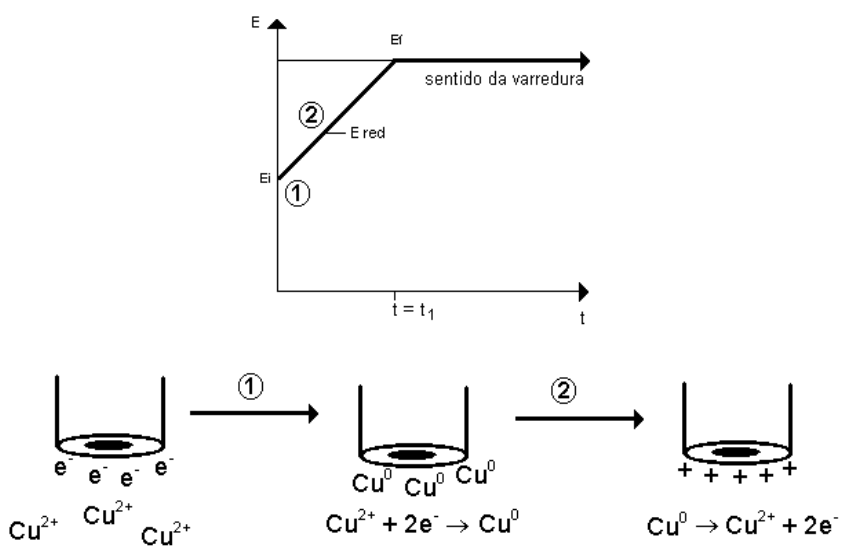

Figura 4. Representação esquemática da etapa anódica na VCRA utilizando EPC-AH como eletrodo de trabalho

Como pode ser observado, o cobre(II) em solução (etapa 1) sofre redução na superfície do eletrodo devido à imposição do potencial $\mathrm{E}_{\mathrm{i}}$. Em seguida, o potencial é varrido no sentido catódico e o cobre metálico é oxidado (etapa 2) ao alcançar o potencial de re-oxidação. A presença de $\mathrm{AH}$ na pasta de carbono pode atuar como um préconcentrador, mesmo que nenhum potencial seja imposto no eletrodo. 


\section{CARACTERÍSTICAS DOS VOLTAMOGRAMAS OBTIDOS COM EPC-AH EM SOLUÇÕES CONTENDO METAIS}

As características dos voltamogramas cíclicos obtidos com EPCAH na determinação de metais em solução variam de acordo com a composição do eletrodo e do tipo de metal estudado ${ }^{9,41,47}$. Na literatura há várias citações de estudos de pré-concentração de metais na superfície do EPC-AH, em que o tempo de pré-concentração e a quantidade de $\mathrm{AH}$ na pasta são fatores importantes ${ }^{10-12,42-47}$. Nesta revisão, optou-se por destacar três estudos, para fim ilustrativo, com metais em solução, com intuito de mostrar o perfil voltamétrico dos metais na superfície do EPC-AH ${ }^{9,41,47}$.

O primeiro estudo destaca as diferenças entre os voltamogramas do EPC e do EPC-AH em eletrólito suporte, para a determinação de cobre (II) ${ }^{9}$. O segundo relata os efeitos da pré-concentração de chumbo (II) na superfície do EPC-AH ${ }^{47}$. E, por fim, o terceiro mostra os efeitos da variação da concentração de um sal de AH em um EPC-AH na determinação de bismuto (III) e como ocorre o efeito da pré-concentração desse metal na superfície eletródica ${ }^{45}$.

$\mathrm{O}$ voltamograma de um EPC-AH difere do EPC devido ao fato do $\mathrm{AH}$ presente na superfície do eletrodo aumentar o efeito resistivo e por ocorrerem algumas reações redox nas próprias moléculas de $\mathrm{AH}^{47}$. A Figura 5 apresenta dois voltamogramas cíclicos (EPC e EPC$\mathrm{AH})$ obtidos em tampão ácido acético-acetato de sódio $0,1 \mathrm{~mol} \mathrm{~L}^{-1}$ $(\mathrm{pH} 4,4)^{9}$.

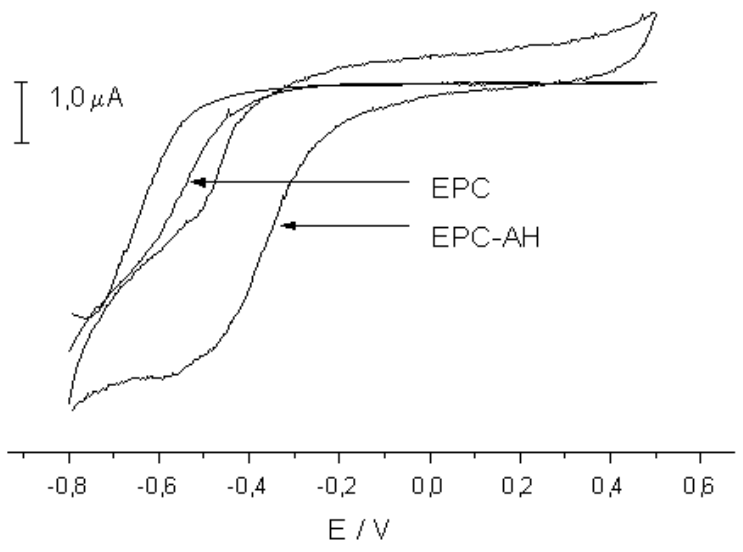

Figura 5. Voltamogramas cíclicos do tampão ácido acético-acetato de sódio $0,1 \mathrm{~mol} \mathrm{~L}^{-1}(\mathrm{pH} 4,4)$ com o EPC e EPC-AH. Velocidade de varredura $=10$ $\mathrm{mV} \mathrm{s} \mathrm{s}^{-1}$. Eletrodo de referência $\mathrm{Ag} / \mathrm{AgCl}$ (fonte: ref. 9)

Pode-se verificar que os voltamogramas apresentaram características diferentes para ambos os eletrodos. A varredura de uma solução de cobre (II) $1,6 \times 10^{-5} \mathrm{~mol} \mathrm{~L}^{-1}$ em tampão ácido acético-acetato de sódio $0,1 \mathrm{~mol} \mathrm{~L}^{-1}(\mathrm{pH} 4,4)$, com tempo de pré-concentração igual a 5 min $(-0,8 \mathrm{~V})$, está representada na Figura 6.

Pode-se observar pela Figura 6 que o pico anódico do cobre (II) é maior com o EPC-AH que com o EPC. Estes resultados são um indicativo de que os $\mathrm{AH}$ presentes na superfície do eletrodo modificado intensificam o fenômeno de acumulação do cobre, significando um ganho de corrente de aproximadamente $6 \mu \mathrm{A}$ (30\%) para essas condições. Além disso, verificou-se com o EPC-AH um deslocamento no potencial de oxidação para regiões menos positivas 9 .

Para um EPC-AH modificado com $4 \%$ de AH (m/m) observouse que a pré-concentração de chumbo (II) obedece uma equação do tipo exponencial ${ }^{47}$. O experimento que comprovou esse resultado foi realizado com pré-concentração de chumbo na superfície do eletro- do, agitando uma solução de chumbo (II) $1,0 \times 10^{-6} \mathrm{~mol} \mathrm{~L}^{-1}$, $\mathrm{pH} \mathrm{4,4}$ (tampão acetato $0,1 \mathrm{~mol} \mathrm{~L}^{-1}$ ) durante $45 \mathrm{~min}$. A Figura 7 mostra os voltamogramas obtidos com o EPC e o EPC-AH.

O EPC-AH mostrou-se mais sensível, pois a corrente de pico anódica é maior, quando comparada à obtida por EPC. Assim, os AH contribuem para o efeito de pré-concentração de chumbo na superfície eletródica. A Figura 8 mostra como a intensidade da corrente de pico varia com o tempo de pré-concentração até $10 \mathrm{~min}$.

A corrente varia exponencialmente com o tempo de pré-concentração, até $10 \mathrm{~min}$. Segundo os autores, após esse tempo essa relação não é mais respeitada ${ }^{47}$. A equação que rege o sistema é descrita por $I_{p}=112,76 \times \exp (t / 2,51)$.

Resultados obtidos por Wang ${ }^{45}$ em estudos de determinação de bismuto (III) demonstraram que o aumento da concentração de humato de sódio (sal de $\mathrm{AH}$ ) na superfície do eletrodo aumenta a corrente de fundo. $\mathrm{O}$ efeito da pré-concentração de bismuto na superfície do EPC-AH também foi estudado nesse trabalho. No caso do bismuto (III), os autores observaram uma relação linear entre a corrente de pico anódica e o tempo de pré-concentração até 30 min. Após esse tempo, a relação não é mais linear.

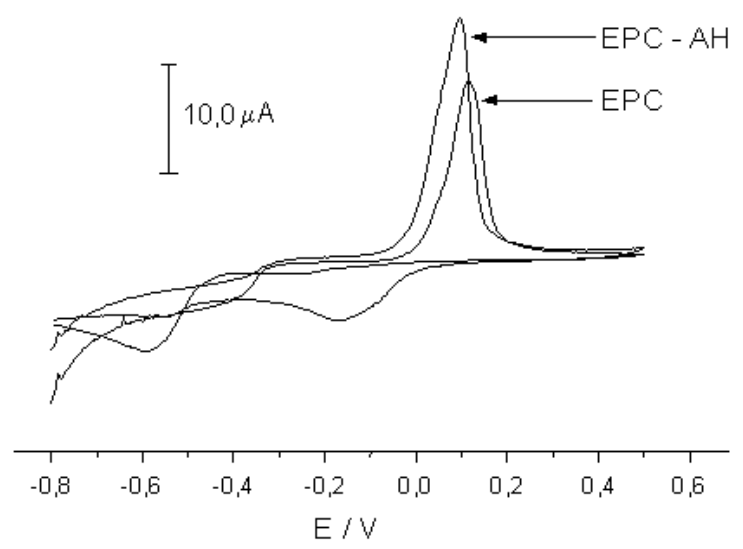

Figura 6. Voltamogramas cíclicos de uma solução de cobre (II) $1,6 \times 10^{-5}$ mol $L^{-1}$ em tampão ácido acético-acetato de sódio $0,1 \mathrm{~mol} \mathrm{~L}^{-1}(\mathrm{pH} \mathrm{4,4)} \mathrm{com}$ o EPC e EPC-AH. Eletrodo de referência Ag/AgCl. Tempo de préconcentração $=5 \min (-0,8 \mathrm{~V})$. Velocidade de varredura $=10 \mathrm{mV} \mathrm{s}^{-1}$ (fonte: ref. 9)

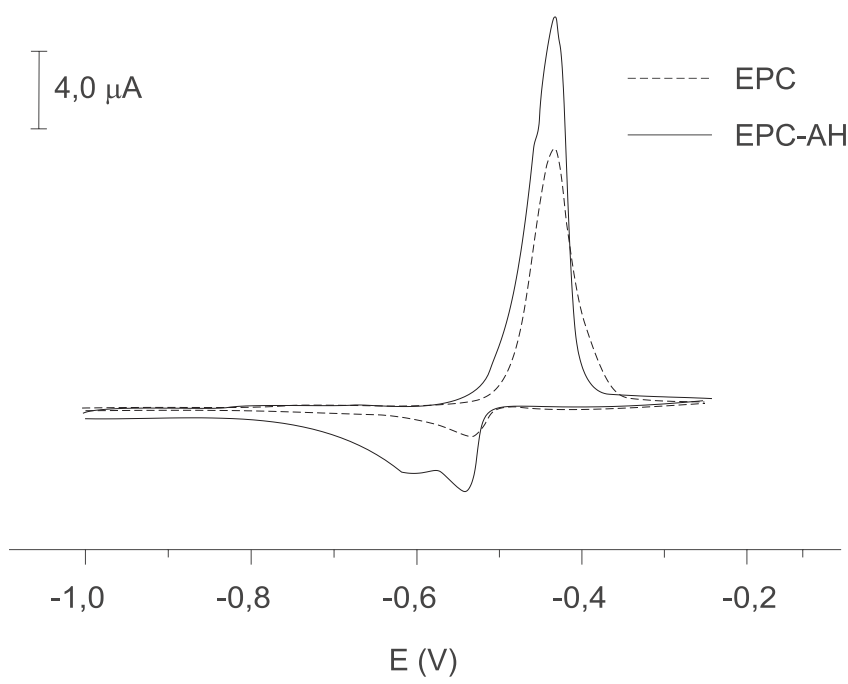

Figura 7. Voltamogramas cíclicos para o chumbo (II) utilizando EPC e o EPC-AH. Tempo de pré-concentração: 45 min (fonte: ref. 47) 


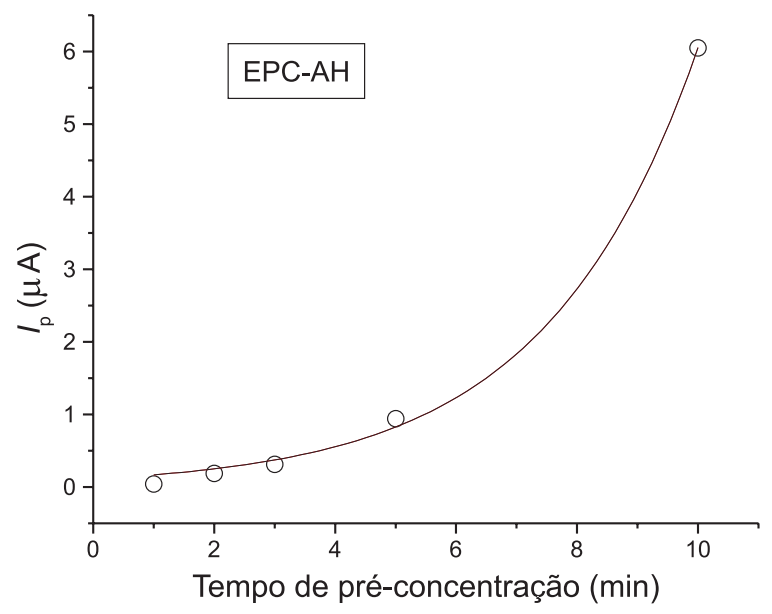

Figura 8. Corrente anódica obtida com o EPC-AH em função do tempo de

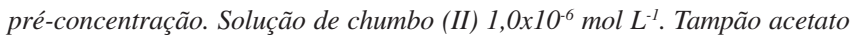

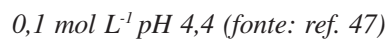

A partir dos exemplos citados, observam-se algumas diferenças no comportamento voltamétrico envolvendo diferentes metais e composição de AH em um EPC-AH ${ }^{9,45,47}$. No entanto, nesses trabalhos é possível identificar a interação entre os $\mathrm{AH}$ e os metais analisados, denotando que o EPC-AH é um bom sensor para determinação voltamétrica de metais em solução.

A Tabela 1 apresenta os estudos envolvendo EPC-AH em ordem cronológica.

\section{ALGUNS ASPECTOS TEÓRICOS DA INTERAÇÃO AH- METAL EM EPC-AH}

A análise teórica e a determinação da constante de estabilidade de um metal complexado aos AH de um EPC-AH foi proposta por Wang e colaboradores ${ }^{42}$. Os autores determinaram a constante de estabilidade do cobre(II) complexado com os AH. A teoria baseia-se em determinar alguns parâmetros via VC e utilizá-los nos cálculos da constante.

Quando os AH presentes no EPC-AH são imersos em uma solução contendo íons metálicos $\mathrm{M}^{\mathrm{n}+}$, pode ocorrer a complexação desses íons com os grupos funcionais (L) presentes nos AH. A imobilização do íon pelo ligante dos AH (L-EPC) pode ser representada pela seguinte reação de complexação:
$\mathrm{M}^{\mathrm{n}+}+\mathrm{L}-\mathrm{EPC} \rightarrow \mathrm{ML}^{\mathrm{n}+}-\mathrm{EPC}$

As cargas do ligante L estão omitidas e as cargas do complexo na Equação 1 não são exatas, para simplificação do cálculo. Assim, a constante de estabilidade pode ser definida como:

$k=\frac{\left[M L^{n+}\right]}{\left[M^{n+}\right][L]}$

Quando se realiza a pré-concentração de $\mathrm{M}^{\mathrm{n}+}$ pode ocorrer saturação dos íons metálicos ligados ao $\mathrm{AH}$ na superfície do eletrodo, dada por $\left[\mathrm{ML}^{\mathrm{n}+}\right]_{\text {sat }}$. Esse processo pode ser acompanhado por um gráfico $I_{\mathrm{p}}$ x $T_{\mathrm{a}}$, onde $I_{\mathrm{p}}$ é a corrente de pico anódica ou catódica e $T_{\mathrm{a}}$ é o tempo de pré-concentração. Após a saturação, pode-se varrer o potencial em uma solução contendo apenas eletrólito suporte, de tal modo a reduzir o metal complexado na superfície do eletrodo de $\mathrm{M}^{\mathrm{n+}}$ a M. No caso da VC, quando ocorre a inversão da varredura procede-se à oxidação de $\mathrm{M}$. A equação desse processo pode ser descrita como:

$\mathrm{ML}^{\mathrm{n}+}-\mathrm{EPC}+\mathrm{ne}^{-} \rightarrow \mathrm{M}+\mathrm{L}-\mathrm{EPC}$

Assim, a corrente de pico catódica $\left(I_{\mathrm{pc}}\right)$ e a corrente de pico anódica $\left(I_{\mathrm{pa}}\right)$ são dadas pelas seguintes equações:

$I_{p c}=\frac{\alpha n^{2} F^{2} S\left[M L^{n+}\right] v}{4 R T}$

$I_{p a}=\frac{\beta n^{2} F^{2} S[M] v}{4 R T}$

onde, $\alpha$ e $\beta$ são os coeficientes de transferência eletrônica de redução e oxidação, respectivamente; $n$ é o número de elétrons envolvido; $F$ é a constante de Faraday; $v$ é a velocidade de varredura $\left(\mathrm{V} \mathrm{s}^{-1}\right)$; $S$ é a área do eletrodo $\left(\mathrm{cm}^{2}\right) ; R$ é a constante universal dos gases ideais e $T$ é a temperatura $(\mathrm{K})$.

Antes de varrer o potencial em certo volume $V\left(\mathrm{dm}^{3}\right)$ de eletrólito suporte, ao colocar o eletrodo em contato com eletrólito haverá uma tendência de migração do metal complexado $\mathrm{M}^{\mathrm{n}+}$ para a solução eletrolítica. $\mathrm{O}$ equilíbrio será atingido em um tempo $t_{\mathrm{eq}}$, que é o tempo de contato entre a superfície do eletrodo e o eletrólito necessário para que o equilíbrio seja atingido. A concentração das espécies em equilíbrio complexada na superfície do eletrodo $\left[\mathrm{ML}^{\mathrm{n}+}\right]_{\mathrm{eq}}$ pode ser facilmente obtida pela Equação 1, em $t_{\text {eq }}$. A concentração total do

Tabela 1. Cronologia dos estudos envolvendo EPC-AH

\begin{tabular}{|c|c|c|c|}
\hline Autores & Metais & Estudo & Ref. \\
\hline Wang (1992) & $\mathrm{Cu}(\mathrm{II})$ & Determinação & 37 \\
\hline Navrátilová e Kula (1992) & $\mathrm{Hg}(\mathrm{II})$ & Determinação & 38 \\
\hline Navrátilová e Kula (1993) & $\mathrm{Cu}(\mathrm{II}), \mathrm{Hg}(\mathrm{II}), \mathrm{Ag}(\mathrm{I}), \mathrm{Zn}(\mathrm{II})$ & Complexação AH-metal & 39 \\
\hline Jeong et al. (1994) & $\mathrm{Cu}(\mathrm{II})$ & Pré-concentração para quantificação & 40 \\
\hline Wang et al. (1997) & $\mathrm{Bi}(\mathrm{III})$ & Determinação & 45 \\
\hline Lu et al. (1997) & $\mathrm{Cu}(\mathrm{II})$ & Interação AH-metal & 48 \\
\hline Wang e Li (1998) & $\mathrm{Hg}(\mathrm{I}), \mathrm{Hg}(\mathrm{II})$ & Interação AH-metal & 44 \\
\hline Wang et al. (1998) & $\mathrm{Au}(\mathrm{III})$ & Determinação & 43 \\
\hline Wang et al. (1998) & $\mathrm{Cu}(\mathrm{II})$ & Constante de estabilidade $\mathrm{AH}$-metal & 42 \\
\hline Ghantous et al. (1998) & $\mathrm{Cu}(\mathrm{II}), \mathrm{Cd}(\mathrm{II}), \mathrm{Pb}(\mathrm{II}), \mathrm{U}(\mathrm{IV})$ & Membranas modificadas & 49 \\
\hline Sun et al. (1999) & $\mathrm{Pd}(\mathrm{II})$ & Determinação & 50 \\
\hline Silva $(2000)$ & $\mathrm{Cu}(\mathrm{II})$ & Interação e quantificação & 41 \\
\hline Silva et al. (2002) & $\mathrm{Cu}(\mathrm{II})$ & Interação AH-metal & 10 \\
\hline Crespilho et al. (2002) & $\mathrm{Cu}(\mathrm{II})$ & Determinação em aguardente & 9 \\
\hline
\end{tabular}


ligante [L] pode ser obtida pela equação:

$[\mathrm{L}]=\left[\mathrm{ML}^{\mathrm{n}+}\right]_{\mathrm{sat}}-\left[\mathrm{ML}^{\mathrm{n}+}\right]_{\mathrm{sol}}$

Tendo o valor de [L], o volume $(V)$ do eletrólito suporte utilizado na VC e o valor da área do eletrodo (S), pode-se calcular a concentração de equilíbrio do íon $\mathrm{M}^{\mathrm{n}+}$ pela seguinte equação:

$$
\left[M^{n+}\right]_{e q}=\frac{[L] S}{V}
$$

Assim, pode-se obter o valor da constante de estabilidade pela equação:

$K=\frac{\left[M L^{n+}\right]_{e q} V}{S[A]_{e q}^{2}}$

Para complexos com número de coordenação maior que 1 a Equação 8 pode ser adaptada ${ }^{42}$.

\section{CONSIDERAÇÕES FINAIS E ANÁLISE CRÍTICA}

Observando os trabalhos já realizados utilizando EPC-AH, há uma tendência na diminuição da porcentagem de $\mathrm{AH}$ como agente modificador em eletrodos de pasta de carbono. Essa tendência surge com o intuito de diminuir a resistividade, mantendo boa sensibilidade do eletrodo. A homogeneização em meio alcalino do AH com o pó de grafite talvez seja uma das melhores maneiras de preparação da pasta, porém, ainda se faz necessário desenvolver métodos rápidos de preparação de eletrodos sem que a estrutura dos AH seja alterada em demasia.

Ao adicionar os AH em uma solução alcalina e depois liofilizálos, ocorre a formação de humatos. Portanto, em muitos trabalhos registrados na literatura, sais de $\mathrm{AH}$ foram utilizados na preparação de EPC-AH. Assim, esses eletrodos poderiam ser melhor classificados como sendo eletrodos modificados com sal de AH. Esse fato não implica diretamente na qualidade dos resultados obtidos até o presente momento, tendo em vista o grande avanço na otimização de EPC-AH nos últimos tempos.

É de se considerar, também, que vários metais ainda não foram analisados e que possivelmente ter-se-á vários trabalhos publicados envolvendo diferentes tipos de metais e EPC-AH. Nestes primeiros 11 anos da existência do EPC-AH, foi possível verificar que a maioria dos trabalhos convergem para o mesmo enfoque: a determinação de metais e o estudo da interação AH-metal em meio aquoso. Porém, alguns compostos orgânicos podem ser estudados via EPC-AH ${ }^{11,12}$, abrindo novos caminhos para a utilização desses eletrodos.

\section{AGRADECIMENTOS}

Os autores agradecem à CAPES, CNPq e FAPESP pelo suporte financeiro dado aos projetos envolvendo eletrodos modificados.

\section{REFERÊNCIAS}

1. Rieger, P. H.; Electrochemistry, $2^{\text {nd }}$ ed., Chapman \& Hall: New York, 1994.

2. Fry, A. J.; Synthetic Organic Electrochemistry, $1^{\text {th }}$ ed., Publishers: New York, 1972.
3. Souza, D.; Machado, S. A. S.; Avaca, L. A.; Quim. Nova 2003, $26,81$.

4. Demircigil, B. T.; Uslu, B.; Özkan, Y.; Özkan, S. A.; Sentürk, Z.; Electroanalysis 2003, 15, 230.

5. Prasad, B. B.; Arora, B.; Electroanalysis 2003, 15, 108.

6. Mlakar, M.; Electroanalysis 2003, 15, 27.

7. Reguera, C.; Ortiz, M. C.; Arcos, M. J.; Electroanalysis 2002, 14, 1699.

8. Ferancová, A.; Korgová, E.; Labuda, J.; Zima, J.; Barek, J.; Electroanalysis 2002, 14, 1668.

9. Crespilho, F. N.; Messias, R. A.; Rezende, M. O. O.; An. Assoc. Bras. Quím. 2002, 51, 63 .

10. Silva, W. T. L.; Thobie-Gautier, Cv.; Rezende, M. O. O.; El Murr, N.; Electroanalysis 2002, 14, 71 .

11. Crespilho, F. N.; Silva, W. T. L.; Rezende, M. O. O.; An. Assoc. Bras. Quím. 2003, 52, 58.

12. Crespilho, F. N.; Airoldi, F. P. S.; Silva, W. T.; Rezende, M. O. O.; Resumos do $15^{\circ}$ Congresso da Sociedade Ibero-Americana de Eletroquímica, Évora, Portugal, 2002.

13. Adams, R. N.; Anal. Chem. 1958, 30,1576.

14. Oslon, C.; Adams, R. N.; Anal. Chim. Acta 1960, $22,582$.

15. Oslon, C.; Adams, R. N.; Anal. Chim. Acta 1963, 29, 358.

16. Kuwana, T.; French, W. G.; Anal. Chem. 1964, 36, 241.

17. Schultz, F. A.; Kuwana, T.; J. Electroanal. Chem. 1965, 10, 95.

18. http://www.sciencedirect.com, acessada em Março 2003.

19. Cheek, G. T.; Nelson, R. F.; Anal. Lett. 1978, 11, 393.

20. Ravichandran, K.; Baldwin, R. P.; J. Electroanal. Chem. 1981, 126, 293.

21. Vasilescu, A.; Noguer, T.; Andreescu, S.; Calas-Blanchard, C.; Bala, C.; Marty, J. L.; Electroanal. Chem. 2003, 59, 751.

22. Kalcher, K.; Kauffmann, J. M.; Wang, J.; Svancara, I.; Vytras, K.; Neuhold, C.; Yang, Z.; Electroanalysis 1995, 7, 5.

23. Kalcher, K.; Grabec, I.; Raber, G.; Cai, X.; Tavcar, G.; Ogorevc, B.; J. Electroanal. Chem. 1995, 386, 149.

24. Yabuki, S.; Mizutami, F.; Biosens. Bioelectron. 1995, 10, 353.

25. Razumas, V.; Kazlauskaité, J.; Vidziunaité R.; Bioelectroch. Bioener. 1996, 39, 139.

26. Stadlober, M.; Kalcher, K.; Raber, G.; Neuhold, C.; Talanta 1996, 43,1915.

27. Lupetti, K. O.; Fatibello-Filho, O.; Quim. Nova 2003, 26, 39.

28. El Murr, N.; Kerkeni, M.; Sellami, A.; Bem Taarit, Y.; J. Electroanal. Chem. 1988, 246, 461.

29. Stevenson, F. J.; Humic Substances in the Environment, $1^{\text {th }}$ ed., Marcel Decker: New York, 1972.

30. Schulten, H. R.; Schnitzer, M. A.; Naturwissenschaften 1993, 80, 29.

31. Sein Jr., L. T.; Varnum, J. M.; Jansen, S. A.; Environ. Sci. Technol. 1999, $33,546$.

32. Sargentini Jr., E.; Rocha, J. C.; Rosa, A. H.; Zara, L. K.; Santos, A.; Quim. Nova 2001, 24, 339.

33. Senesi, N.; Sposito, G.; Martin, J. D.; Sci. Total Environ. 1986, 55, 351.

34. Barros, M. C. P.; Paula, J. R.; Rezende, M. O. O.; Quim. Nova 1994, 17, 376.

35. Landegraf, M. D.; Rezende, M. O. O.; Anal. Chim. Acta 1998, 368, 155.

36. http://www.ihss.gatech.edu, acessada em Março 2003.

37. Wang, J.; Naser, D.; Darnall, D.; Gardea-Torresdey, J.; Electroanalysis 1992, 4, 71 .

38. Navratilova, Z.; Kula, P.; Electroanalysis 1992, 4, 683.

39. Navratilova, Z.; Kula, P.; Anal. Chim. Acta 1993, 273, 305.

40. Jeong, E-D.; Won, M-S.; Shim, Y-B.; Electroanalysis 1994, 6, 887.

41. Silva, W. T. L.; Tese de Doutorado, Universidade de São Paulo, Brasil, 2000.

42. Wang, C.; Zhu, B.; Li, H.; Electroanalysis 1999, 11, 183.

43. Wang, C.; Zang, H.; Sun, Y.; Li, H.; Anal. Chim. Acta 1998, 361, 133.

44. Wang, C.; Zhu, B.; Li, H.; Electroanalysis 1998, 10, 44.

45. Wang, C.; Sun, Q.; Li, H.; Electroanalysis 1997, 9, 645.

46. Bond, A. M.; Modern Polarographic Methods in Analytical Chemistry, $1^{\text {th }}$ ed., Marcel Dekker Inc: New York, 1980.

47. Crespilho, F. N.; Messias, R. A.; Rezende, M. O. O.; Resumos da $25^{a}$ Reunião Anual da Sociedade Brasileira de Química, Poços de Caldas, Brasil, 2002.

48. Lu, X.; Yang, X.; Chen, Z.; Electroanalysis 1997, 9, 1278.

49. Ghantous, L.; Lojou, E.; Bianco, P.; Electroanalysis 1998, 10, 1249.

50. Sun, Q. Y.; Wang, C. M.; Li, L. X.; Li, H. L.; Fresenius J. Anal. Chem. 1999, 363, 114. 